\title{
A Novel Modified-Curcumin Promotes Resolvin-Like Activity and Reduces Bone Loss in Diabetes-Induced Experimental Periodontitis
}

Jie Deng, (D) Lorne M Golub, ${ }^{2}$ Hsi-Ming Lee, ${ }^{2}$ Veena Raja, ${ }^{2}$ Francis Johnson, (iD ${ }^{3}$ Allan Kucine, ${ }^{4}$ Wonsae Lee, ${ }^{5}$ Tian-Min Xu,' Ying Gu ${ }^{6}$

'Department of Orthodontics, Peking University School and Hospital of Stomatology \& National Clinical Research Center for Oral Diseases \& National Engineering Laboratory for Digital and Material Technology of Stomatology \& Beijing Key Laboratory of Digital Stomatology, Beijing, 10008I, People's Republic of China; ${ }^{2}$ Department of Oral Biology and Pathology, School of Dental Medicine, Stony Brook University, Stony Brook, NY, I I794, USA; ${ }^{3}$ Department of Chemistry and Pharmacological Sciences, School of Medicine, Stony Brook

University, Stony Brook, NY, I I794, USA;

${ }^{4}$ Department of Oral \& Maxillofacial Surgery, School of Dental Medicine, Stony Brook University, Stony Brook, NY, I I794, USA; ${ }^{5}$ Department of Biomedical Engineering, Stony Brook University, Stony Brook, NY, I I794, USA; ${ }^{6}$ Department of General Dentistry, School of Dental Medicine, Stony Brook University, Stony Brook, NY, I I 794, USA

Correspondence: Jie Deng Department of Orthodontics, Peking University School and Hospital of

Stomatology \& National Clinical Research Center for Oral Diseases \& National

Engineering Laboratory for Digital and Material Technology of Stomatology \& Beijing Key Laboratory of Digital

Stomatology, 22 Zhongguancun South

Avenue, Haidian District, Beijing, I0008I,

People's Republic of China

Tel +I 63I 632-3172

Fax +I 63I 632-9705

Email jie.deng917@gmail.com

Tian-Min Xu

Email tmxuortho@163.com
Purpose: Clinically, it is challenging to manage diabetic patients with periodontitis. Biochemically, both involve a wide range of inflammatory/collagenolytic conditions which exacerbate each other in a "bi-directional manner." However, standard treatments for this type of periodontitis rely on reducing the bacterial burden and less on controlling hyperinflammation/excessive-collagenolysis. Thus, there is a crucial need for new therapeutic strategies to modulate this excessive host response and to promote enhanced resolution of inflammation. The aim of the current study is to evaluate the impact of a novel chemicallymodified curcumin 2.24 (CMC2.24) on host inflammatory response in diabetic rats.

Methods: Type I diabetes was induced by streptozotocin injection; periodontal breakdown then results as a complication of uncontrolled hyperglycemia. Non-diabetic rats served as controls. CMC2.24, or the vehicle-alone, was administered by oral gavage daily for 3 weeks to the diabetics. Micro-CT was used to analyze morphometric changes and quantify bone loss. MMPs were analyzed by gelatin zymography. Cell function was examined by cell migration assay, and cytokines and resolvins were measured by ELISA.

Results: In this severe inflammatory disease model, administration of the pleiotropic CMC2.24 was found to normalize the excessive accumulation and impaired chemotactic activity of macrophages in peritoneal exudates, significantly decrease MMP-9 and proinflammatory cytokines to near normal levels, and markedly increase resolvin $\mathrm{D}_{1}\left(\mathrm{RvD}_{1}\right)$ levels in the thioglycolate-elicited peritoneal exudates (tPE). Similar effects on MMPs and $\mathrm{RvD}_{1}$ were observed in the non-elicited resident peritoneal washes (rPW). Regarding clinical relevance, CMC2.24 significantly inhibited the loss of alveolar bone height, volume and mineral density (ie, diabetes-induced periodontitis and osteoporosis).

Conclusion: In conclusion, treating hyperglycemic diabetic rats with CMC2.24 (a triketonic phenylaminocarbonyl curcumin) promotes the resolution of local and systemic inflammation, reduces bone loss, in addition to suppressing collagenolytic MMPs and proinflammatory cytokines, suggesting a novel therapeutic strategy for treating periodontitis complicated by other chronic diseases.

Keywords: hyperglycemia, periodontitis, matrix metalloproteinases, inflammation, resolvins, host-modulatory therapy

\section{Introduction}

Interaction of periodontitis and diabetes has long been explored. Patients with both periodontitis and diabetes exhibited higher levels of oral and systemic inflammatory markers than healthy subjects, such as interleukins (IL-1 $\beta$, IL-6), ${ }^{1,2}$ nod-like receptor 
family proteins (NLRP), ${ }^{1}$ and tumor necrosis factor $(\mathrm{TNF}-\alpha){ }^{3}$ Each condition makes the other more severe, linking the two diseases in a "bi-directional manner" as follows. Inflammatory mechanisms during periodontitis damage the tissues supporting the dentition, including gingiva, periodontal ligament, and alveolar bone, eventually leading to tooth loss, but also impact systemic health. ${ }^{4,5}$ The matrix metalloproteinases (MMPs), including the collagenases, MMP-1, MMP-8 and MMP-13, and gelatinases, MMP-2 and MMP-9, when produced in excessive levels (in contrast to their role, at constitutive levels, in physiologic connective tissue turnover), play a major role in the pathogenesis of numerous diseases, including periodontitis and diabetes, even COVID-19. ${ }^{6}$ A recent study indicates that MMP-9, as an immune-based biomarker, is associated with mortality in COVID-19 patients. ${ }^{7}$ Moreover, these MMPs produced locally by inflamed oral tissues, can travel through the circulation and degrade insulin receptors, which contribute to insulin resistance, promote hyperglycemia, and exacerbate the diabetic condition. ${ }^{8,9}$ As a result, diabetes-induced periodontitis, now called the sixth complication of diabetes, ${ }^{10,11}$ is very difficult to manage with traditional-mechanical therapy, ie, scaling and root planing. Therefore, new therapeutics (including MMP-inhibitors), which modulate the excessive host response and promote resolution of inflammation, are urgently needed.

To date, only two MMP-inhibitor medications have been governmentally approved (US Food \& Drug Administration; also regulatory agencies in Canada \& Europe), ie, the novel NON-antimicrobial formulations of the tetracycline, doxycycline, for the treatment for human periodontitis; ${ }^{12,13}$ and a sustained-release formulation of this medication for the treatment of a chronic inflammatory skin disease, acnerosacea. ${ }^{14}$ The mechanism involves, at least in part, the binding of calcium and zinc, by the $\beta$-diketone moiety of this tetracycline (including minocycline and doxycycline) molecule which then inhibits the activity of these metal-dependent, host-derived, neutral proteinases. ${ }^{15,16}$ More recently, additional host-modulating therapy drugs (HMTs) have been developed including resolvins (polyunsaturated fatty acids) and chemically modified curcumins (CMCs). Numerous studies have supported the therapeutic potential of resolvins, such as eicosapentanoic acid (EPA) and docosahexaenoic acid (DHA), in managing chronic diseases. ${ }^{17,18}$ Regarding the latter, a phenylaminocarbonyl tri-ketonic chemically-modified curcumin 2.24 (CMC2.24), as a lead compound, has an enhanced/potent calcium and zinc-binding bioactivity, and has been extensively studied. This novel pleiotropic compound not only inhibits MMPs, ${ }^{19,20}$ but also reduces pro- inflammatory mediators in cell and tissue culture, and in vivo small (rodent) and large (dog) animal models of various inflammatory/collagenolytic disorders (eg, periodontitis, diabetes including impaired wound healing, acute lung disease, arthritis, and cancer). ${ }^{21-26}$

Hence, the rationale of this study is to describe additional novel therapeutic properties of CMC2.24: its ability to induce the production of anti-inflammatory resolvins (eg, Resolvin $\mathrm{D}_{1}, \mathrm{RvD}_{1}$ ), during diabetes, in addition to inhibiting excessive production of destructive MMPs and pro-inflammatory cytokines which prolong chronic inflammation, and to improve the impaired cell function. Thus, the aim was to test the therapeutic potential of CMC2.24 (a) in vivo, in severely hyperglycemic diabetic rats with/ without thioglycolate stimulation in the peritoneal cavity; and (b) in ex-vivo cultured macrophages.

\section{Materials and Methods}

\section{Animal Study}

The animal study was approved by Stony Brook University's Institutional Animal Care and Use Committee (IACUC, \#230617-23), and was performed in strict accordance with the approved protocol. Animals were housed in the Division of Laboratory Animal Resources (DLAR) at Stony Brook University, with care provided by the center's personnel. This facility follows the Animal Welfare Act (USDA enforced), the Public Health Service Act (OLAW enforced), and NY State law (DOH enforced), and is an AAALAC International accredited facility. The Three Rs (3Rs) were reviewed by SBU DLAR and approved. All procedures were conducted at the same location and were overseen by a fully-qualified veterinarian who was also available for general anesthesia, analgesia, routine, surgical care and euthanasia. Thirty-six adult male SpragueDawley rats (Strain code: CD001; Body Weight/Age: 276-300 grams/60-65 days; viral antibody free; Charles River Laboratories International, Inc., Wilmington, MA, USA) were used in two models: the non-elicited and elicited models. Eighteen rats in each model were randomly distributed into three groups: non-diabetic control $(\mathrm{N})$, diabetes (D), and diabetes+CMC2.24 treatment (D $+\mathrm{CMC} 2.24)$ groups ( $\mathrm{n}=6 \mathrm{rats} /$ group $){ }^{27}$ All rats were single housed in proper sterile-filter capped cages, and were given unlimited access to food and water. Type I diabetics was induced by I.V. injection of streptozotocin (STZ, 70mg/kg body weight; ENZO Life Sciences, Inc., 
Plymouth Meeting, PA, USA) through tail vein. The $\mathrm{N}$ rats were injected with $10 \mathrm{mM}$ citrated saline buffer, pH4.5, served as controls. Hyperglycemia were confirmed in urine by using nonenzymatic test-strips (CTMI 4 LN; Cole-Tayler Marketing Inc., Redsa, CA, USA) in all STZ-injected $\mathrm{D}$ and $\mathrm{D}+\mathrm{CMC} 2.24$ rats. CMC2.24 was administered $30 \mathrm{mg} / \mathrm{kg} /$ daily to the D $+\mathrm{CMC} 2.24$ group by oral gavage for 3 weeks. The $\mathrm{N}$ and $\mathrm{D}$ groups were administered vehicle-alone $(1 \mathrm{~mL}$ of a $2 \%$ carboxymethylcellulose suspension) by oral gavage for the same period of time. Each rat has an equal chance of being assigned to the treatment or vehicle-alone.

\section{Non-Elicited Model}

At the end of 3-weeks therapy, all rats (N, D, and D+CMC2.24 groups) were fasted overnight and euthanized by $\mathrm{CO}_{2}$ inhalation. Resident peritoneal wash (rPW, non-elicited) was collected by peritoneal lavage following intraperitoneal injection of $15 \mathrm{~mL}$ of sterile-cold PBS/3mM EDTA.

\section{Elicited Model}

Four days prior to ending of the 3-weeks therapy, all rats in three groups were injected intraperitoneally with $3 \%$ thioglycolate medium (Thermo Fisher Scientific, Waltham, MA, USA) to elicit a macrophage inflammatory response. $^{28}$ The thioglycolate-elicited peritoneal exudates (tPE) were collected as described previously at 3 weeks. ${ }^{28}$ Jawbones were collected for micro-computerized tomography $(\mu \mathrm{CT})$ analysis. Blood was collected by cardiac puncture for analysis of blood glucose and glycated hemoglobin (HbAlc) levels.

\section{Chemical Reagents}

CMC2.24 was synthesized and provided by Chem-Master Intl., Inc. (99.5\% pure, Stony Brook, NY, USA). Carboxymethyl cellulose as placebo was purchased from Sigma Chemical Co. (St. Louis, MO, USA). All cell culture reagents and other chemical reagents were purchased from Thermo Fisher Scientific (Waltham, MA, USA).

\section{Three-Dimensional $\mu$ CT Morphometric Analysis and Quantification of Bone Loss}

In the elicited model, jawbones were fixed and placed in a standardized sample holder. The maxillae were scanned using high-resolution $\mu \mathrm{CT}$ ( $\mu \mathrm{CT} 40$; Scanco Medical, Bassersdorf, Switzerland) at $20.0 \mu \mathrm{m}$ voxel size, followed by reconstruction of the $\mu \mathrm{CT}$ images. Results were analyzed using $\mu \mathrm{CT}$ V6.0 software on the HP open platform (OpenVMS Alpha Version 1.3-1 session manager). Two reference points (bucco-palatal embrasure) were identified on the distal surface of the 1st molar and the mesial surface of the 2 nd molar at the height of contour on coronal sections. The average distance from the cemento-enamel junction to alveolar bone crest (CEJ-ABC) was measured to quantify alveolar bone loss at bucco-palatal sites of the distal surface of the 1st molar and the mesial surface of the 2nd molar. ${ }^{29}$

Volume of interest (VOI) was generated by delineating continuously the contours on the axial planes between the distal root of the 1 st molar and the mesial root of the 2 nd molar. The relative bone VOI was calculated by excluding the root and crown volume from the total VOI, then using a bone volume fraction [bone volume/total volume (BV/ $\mathrm{TV})$ ] to obtain the volume of mineralized bone per unit volume of the sample, based on counting voxels. ${ }^{29}$ In addition, bone mineral density (BMD) was calculated by mean voxel value in units of hydroxyapatite density (mg $\left.\mathrm{HA} / \mathrm{cm}^{3}\right){ }^{30}$

\section{Resident Peritoneal Washes (rPW) and Thioglycolate-Elicited Peritoneal Exudates (tPE)}

The rPW and tPE were collected and centrifuged at $1000 \mathrm{rpm}$ for $5 \mathrm{~min}, 4^{\circ} \mathrm{C}$, to separate supernatant cellfree peritoneal fractions (CFPFs) and the mixed cells in the peritoneal cavity. The CFPFs were analyzed for MMP2 and MMP-9 by gelatin zymography. Peritoneal monocytes/macrophages $(\mathrm{M} \varphi \mathrm{s})$ were isolated and purified from the mixed cells.

\section{Gelatin Zymography}

Assays for MMP-2 (pro-form: $72 \mathrm{kDa}$; active-form: 62 $\mathrm{kDa}$ ) and MMP-9 (pro-form: $92 \mathrm{kDa}$; active-form: 82 $\mathrm{kDa}$ ) in the CFPFs from rPW and tPE in non-elicited and elicited models were conducted by gelatin zymography, as described previously. ${ }^{21}$

\section{Cell Culture Studies (ex-vivo)}

The peritoneal mixed cells from rPW and tPE were resuspended in PBS/3mM EDTA. The cell suspension was layered onto Lymphoprep ${ }^{\mathrm{TM}}$ (Accurate Chemical \& Scientific Corporation, Westbury, NY, USA) at a ratio of 2 to $1-1.5(\mathrm{v} / \mathrm{v})$ then centrifuged at $1800 \mathrm{rpm}$ for $30 \mathrm{~min}$ at 
$25^{\circ} \mathrm{C}$. Peritoneal $\mathrm{M} \varphi \mathrm{s}$ were isolated/purified by the density gradient centrifugation and adherence as described previously. ${ }^{31}$ The non-elicited and elicited M $\varphi$ s were counted and cultured in serum-free media (Thermo Fisher Scientific Inc., Boston, MA, USA) in 24-well plates for 18 hours (exvivo). Each well contained $10^{6}$ cells $/ \mathrm{mL}$, supplemented with 100units $/ \mathrm{mL}$ penicillin and $100 \mu \mathrm{g} / \mathrm{mL}$ streptomycin, in a humidified atmosphere of $5 \% \mathrm{CO}_{2}$ and $95 \%$ air at $37^{\circ} \mathrm{C}$.

\section{Cell Migration Assay}

The elicited peritoneal $\mathrm{M} \varphi \mathrm{s}$ were harvested and directly counted by $\mathrm{TC} 20^{\mathrm{TM}}$ Automated Cell Counter (Bio-Rad Laboratories, Inc. USA). For cell migration/chemotaxis

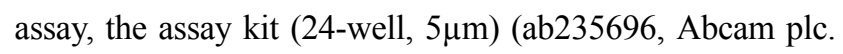
USA) was used with a Boyden chamber, where the cells migrate through a semi-permeable membrane under different stimuli. We used culture medium from LPS-stimulated macrophage as chemoattractant for macrophage migration in the assay. Cell migration was analyzed directly by reading fluorescence $(E x / E m=530 / 590 \mathrm{~nm})$ in a plate reader and was performed according to manufacturer's instructions.

\section{ELISA Assay}

The supernatants from cell culture medium (ex-vivo) of nonelicited and elicited peritoneal $\mathrm{M} \varphi \mathrm{s}$ were analyzed for three pro-inflammatory cytokines: IL- $1 \beta$, IL-6, and TNF- $\alpha$ (R\&D Systems, Inc., Minneapolis, MN, USA), and three resolvins: $\mathrm{RvD}_{1}$ (Cayman Chemical., Ann Arbor, Michigan, USA), Resolvin $\mathrm{E}_{1}\left(\mathrm{RvE}_{1}\right)$ (MyBioSource, Inc., San Diego, CA, USA), and Lipoxin $\mathrm{A}_{4}\left(\mathrm{LxA}_{4}\right)$ (Neogen Corporation, Lexington, Kentucky, USA) by ELISA, and performed according to manufacturer's instructions.

\section{Blood Glucose and HbAlc}

Blood was collected by cardiac puncture for analysis of blood glucose (One Touch Ultra Glucometer, Johnson \& Johnson, NJ, USA) and glycated hemoglobin (HbAlc) levels (Bayer A1CNow Self check, Sunnyvale, CA, USA) by standard procedures.

\section{Statistical Analysis}

SPSS19.0 (IBM) were used to analyze the data. The levels of MMPs, pro-inflammatory cytokines, resolvins, and bone evaluation for $\mathrm{N}, \mathrm{D}, \mathrm{D}+\mathrm{CMC} 2.24$ groups were determined by analysis of variance (ANOVA), and also by Student's $t$-test (two investigators carried out the data analysis, separately), with $\mathrm{p}<0.05$ taken as statistically significant. Each value represented Mean (n=6/group) \pm
Standard Error (S.E.M.). All experiments were independently performed at least 3 times.

\section{Results}

\section{Peritoneal Macrophage Cell Function}

\section{Cell Accumulation and Chemotactic Activity}

Diabetic rats exhibited an abnormal $77 \%$ increase $(\mathrm{p}<0.05)$ in macrophage accumulation (Figure 1A) and a $24 \%$ reduction $(\mathrm{p}<0.05)$ in chemotactic activity when compared to normal rats during thioglycolate-stimulation (Figure 1B). Both abnormalities were "normalized" by treatment of diabetic rats with $\mathrm{CMC} 2.24$. This treatment significantly reduced macrophage accumulation by $87.6 \%$, and improved cell migration ability by $44.1 \%(\mathrm{p}<0.05)$ (Figure $1 \mathrm{~A}$ and $\mathrm{B})$.

\section{Pro-Inflammatory Cytokines}

Diabetes significantly increased the concentrations of all three pro-inflammatory cytokines (IL-1 $\beta$, IL- 6 and TNF- $\alpha$ ) in the thioglycolate-elicited peritoneal $\mathrm{M} \varphi s$, compared to nondiabetic controls, by 5-folds, 7.2-folds, and 1.8-folds, respectively $(\mathrm{p}<0.05)$ (Figure 1C-E). All three cytokines were reduced to normal levels by the CMC2.24 in vivo treatment (Figure 1C-E). In addition, resident non-elicited peritoneal M $\varphi$ s secreted overall lower levels of IL-1 $\beta$ compared to thioglycolate-elicited peritoneal M $\varphi$ s (Figure 1F), but IL-1 $\beta$ was increased in D group, and treatment with systemically-administered CMC2.24 again dramatically reduced IL- $1 \beta$ by $90 \%$ to normal levels $(\mathrm{p}<0.005)$ (Figure 1F). Also, impaired M $\varphi \mathrm{s}$ were "normalized" by $\mathrm{CMC} 2.24$ therapy. No effect was observed for IL- 6 or TNF- $\alpha$ in the resident M $\varphi$ s.

\section{MMPs}

In the thioglycolate-elicited model, both peritoneal $M \varphi s$ and cell-free peritoneal fractions (CFPFs), from the diabetic rats, exhibited significantly elevated MMP-9 levels. In diabetic rats, pro-MMP-9 levels were increased by $80.4 \%$ in the peritoneal M $\varphi$ s (Figure 2A); similarly, in the CFPFs, the levels of total-MMP-9 were increased in $\mathrm{D}$ group by $60 \%$, compared to non-diabetics $(\mathrm{p}<0.05)$ (Figure 2B). Crucially, in vivo $\mathrm{CMC} 2.24$ treatment resulted in a significant reduction of pro-MMP-9 by $89.4 \%(\mathrm{p}<0.05)$ in the peritoneal $\mathrm{M} \varphi \mathrm{s}$, and by $33 \%$ $(\mathrm{p}<0.05)$ of total-MMP-9 in the CFPFs, respectively, back to normal levels (Figure 2A and B).

The MMP-2 levels were not detected in the elicited peritoneal M $\varphi$, while both pro- and active-MMP-2 were produced in the CFPFs. In vivo CMC2.24 treatment 
A
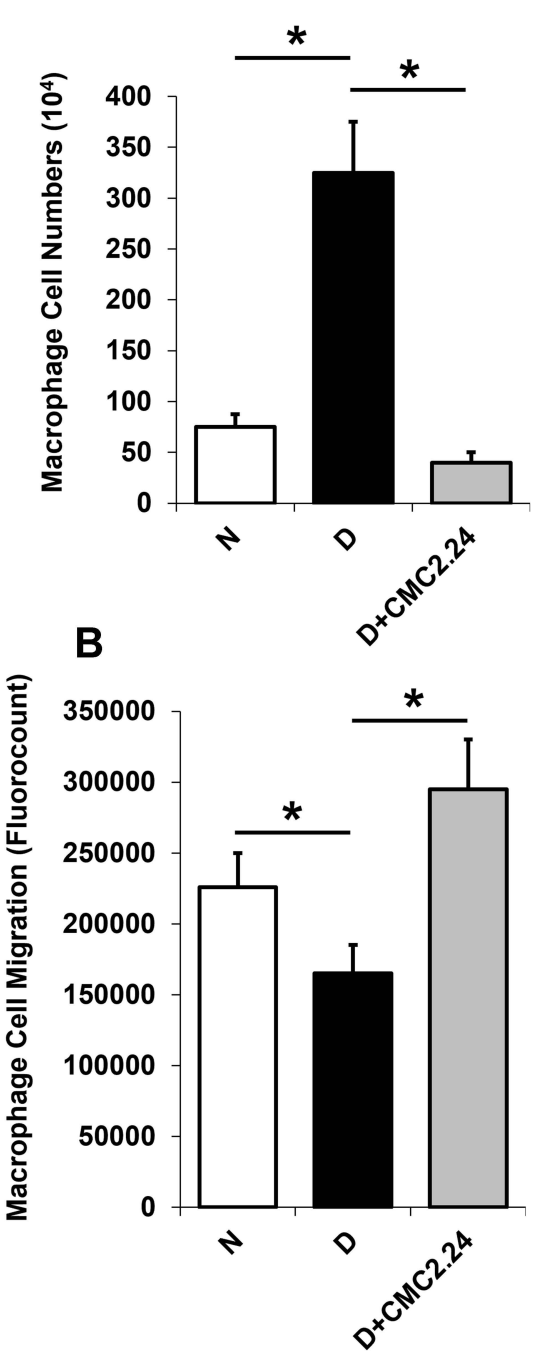

C

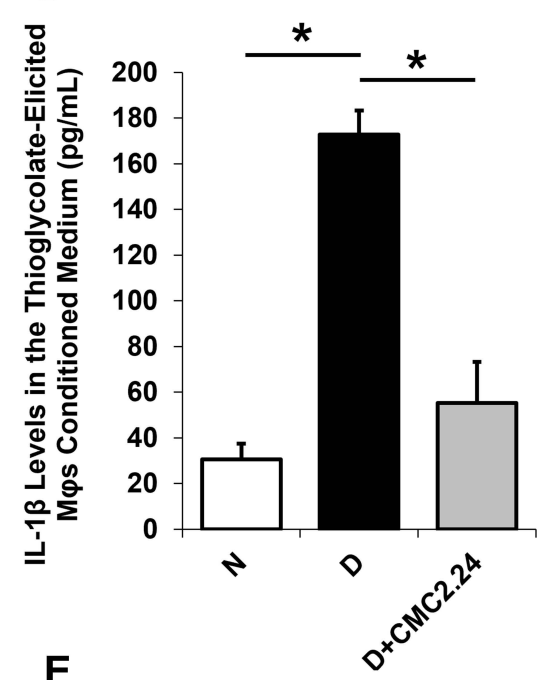

E

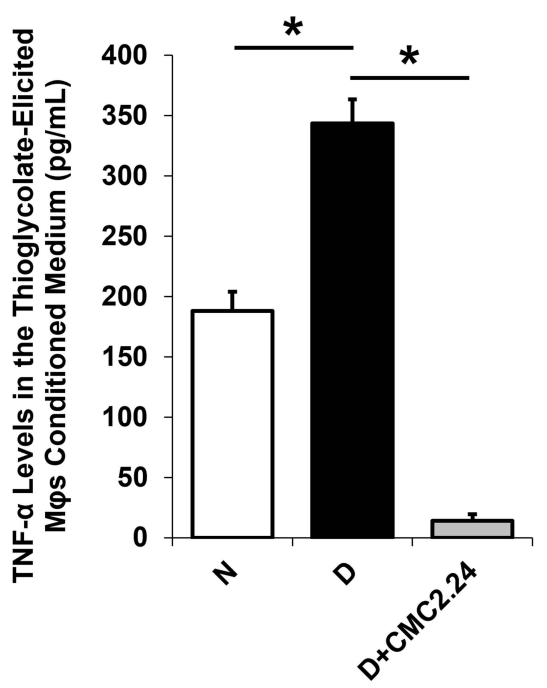

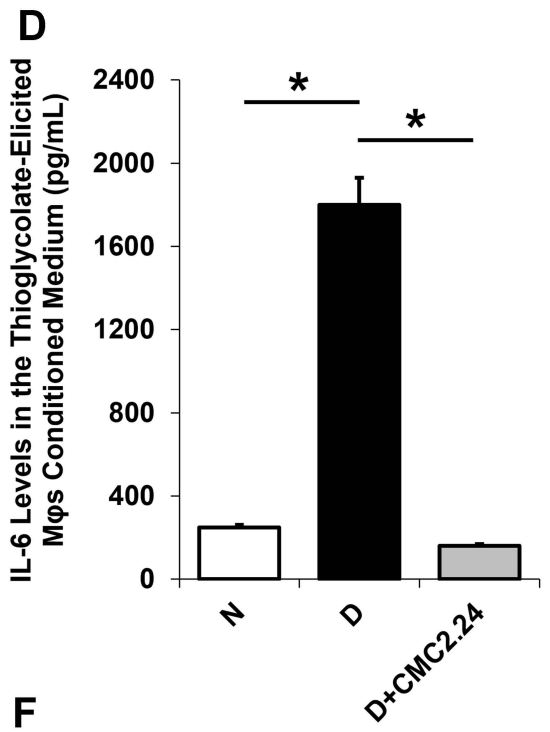

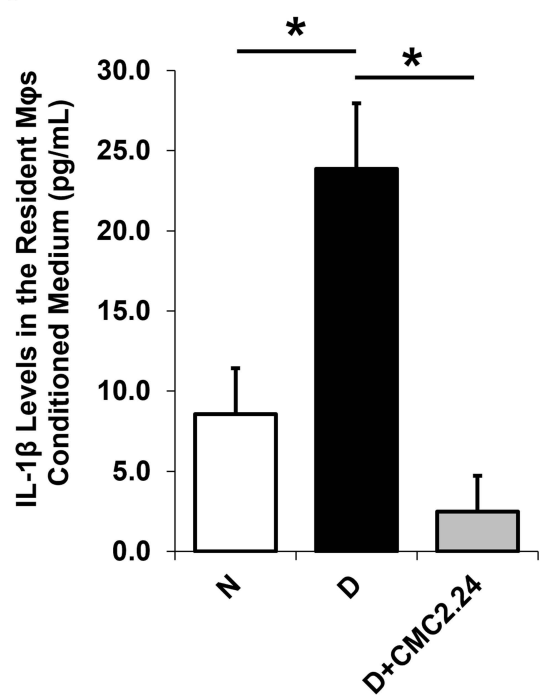

Figure I Effects of in vivo CMC2.24 treatment on peritoneal macrophage (M $\varphi$ ) cell function and pro-inflammatory cytokines in the elicited and non-elicited models of diabetic rat. (A) Cell accumulation was measured by counting of M $\varphi s$ cell numbers. Each column represents $10^{4}$ cells. (B) Chemotactic activity was measured by M $\varphi s$ cell migration (fluorocount). (C-E) IL-I $\beta$, IL-6 and TNF- $\alpha$ levels in the thioglycolate-elicited M $\varphi s$ conditioned media (pg/mL). (F) IL-I $\beta$ levels in the resident non-elicited M $\varphi s$ conditioned medium (pg/mL). Each value represents Mean ( $\mathrm{n}=6 /$ group $) \pm$ Standard Error (S.E.M.). ${ }^{*} \mathrm{p}<0.05$, values were compared between groups at the same time period. Abbreviations: N, normal group; D, diabetic group; D+CMC2.24, diabetes+CMC2.24 treatment group.

tended to reduce this gelatinase to normal levels, but the changes were not significant (data not shown).

In the non-elicited model, D group exhibited significantly increased MMP-9 levels in the CFPFs, including the pro-form $(92 \mathrm{kDa})(\mathrm{p}<0.001)$, and active-form $(82$ $\mathrm{kDa})(\mathrm{p}<0.05)$, as well as the total-MMP-9 ( $<<0.001)$, compared to $\mathrm{N}$ group (Figure $2 \mathrm{C}$ ). The total-MMP-9 in D group was $81 \%$ higher than that in the non-diabetic controls. Treatment with systemically-administered CMC2.24 significantly decreased the pathologically-elevated levels of total-MMP-9 by 47\% ( $p<0.05)$.
Specifically, CMC2.24 treatment decreased the activeMMP-9 significantly ( $p<0.05$ ), compared to D group, and "normalized" this active-form of enzyme efficiently compared to $\mathrm{N}$ group $(\mathrm{p}>0.05)$ (Figure 2C).

Diabetes also increased pro-MMP-2 (72 kDa) by $73 \%$ $(p<0.001)$ in the non-elicited CFPFs, compared to normal rats, whereas CMC2.24 therapy reduced pro-MMP-2 levels by $56 \%(\mathrm{p}<0.005)$, back to normal levels (Figure 2D); the pro-MMP-2 levels in the $\mathrm{N}$ and $\mathrm{D}$ $+\mathrm{CMC} 2.24$ groups were not statistically different $(\mathrm{p}>0.05)$. 

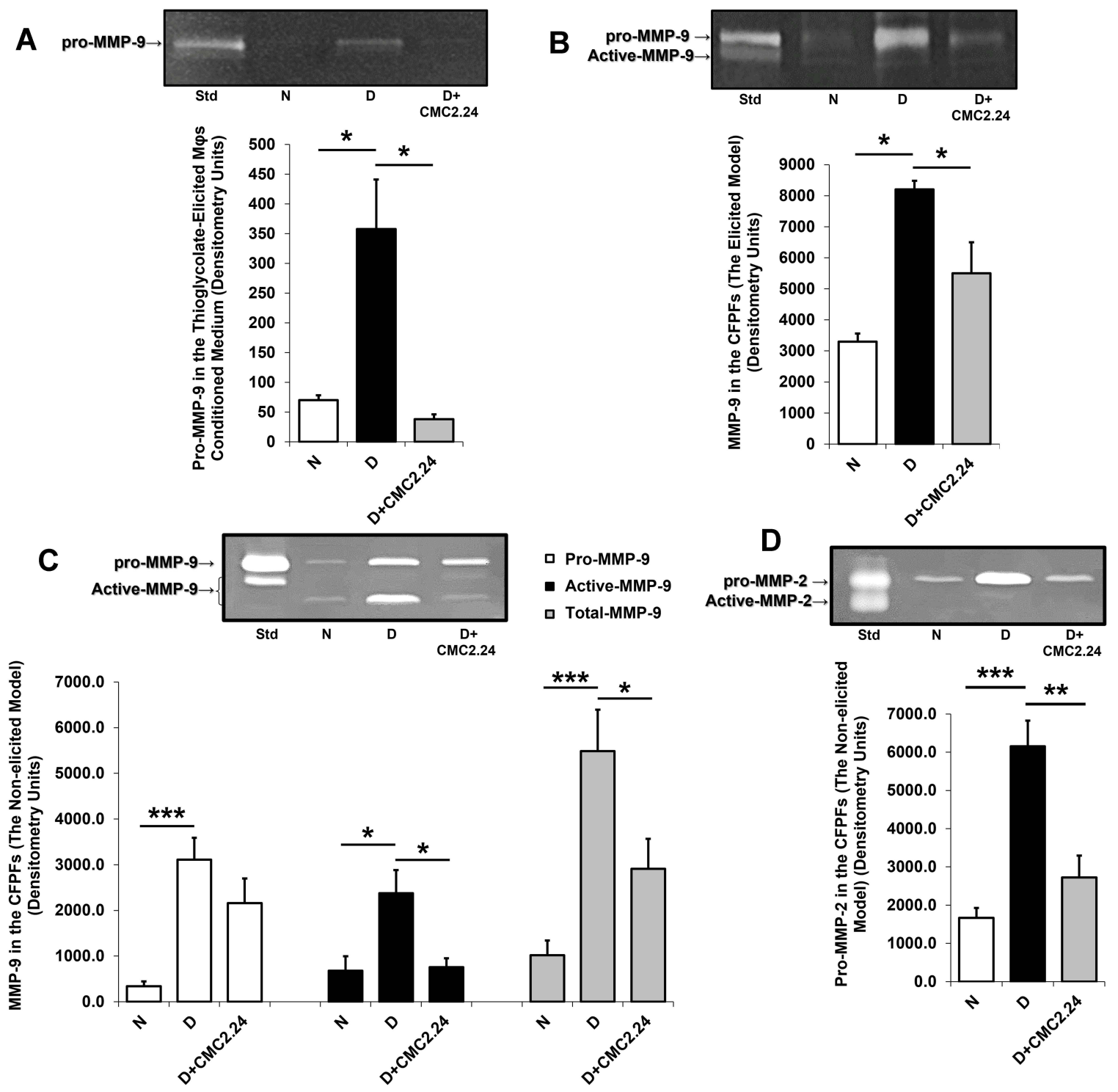

口 Pro-MMP-9

- Active-MMP-9

口 Total-MMP-9
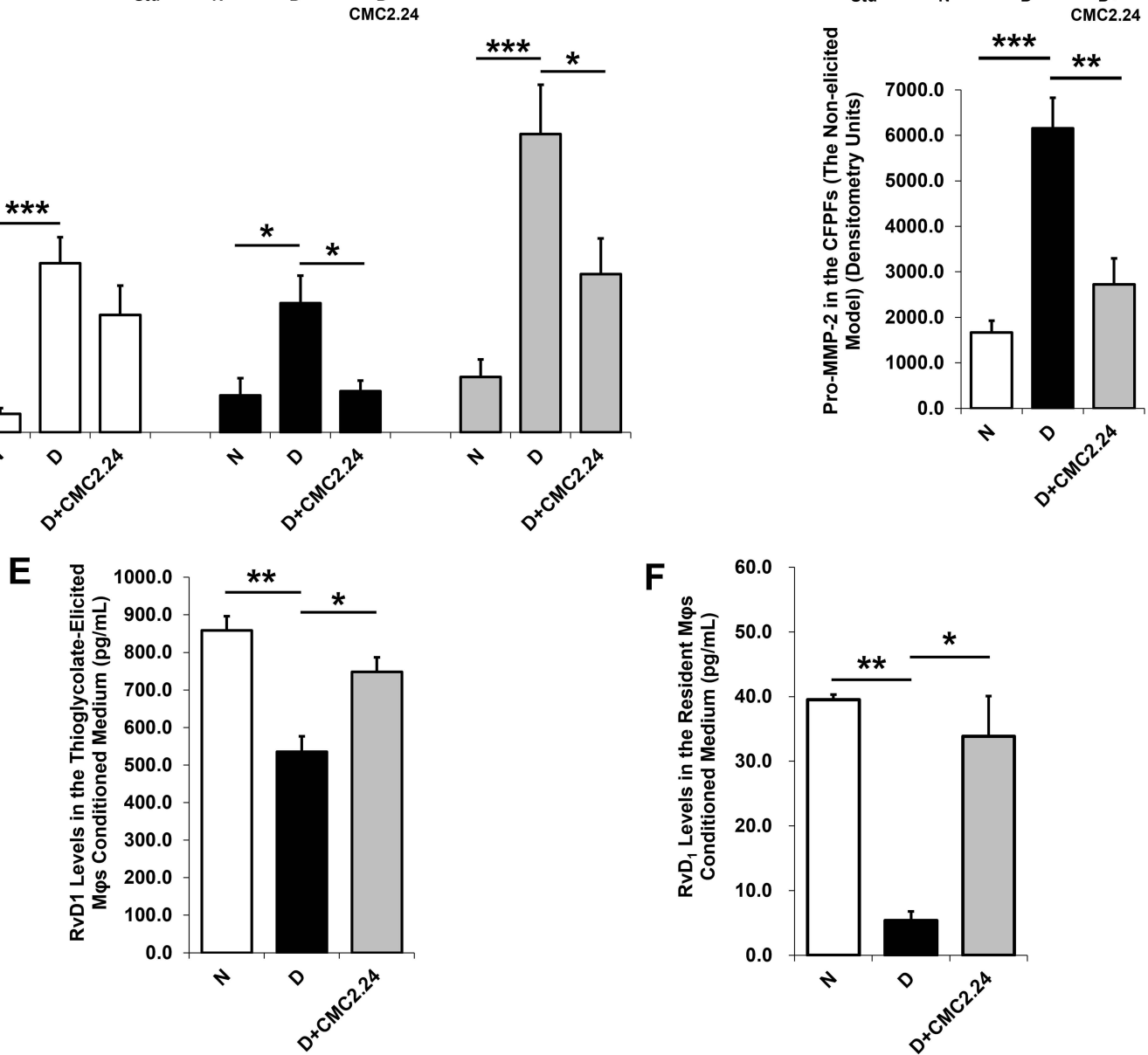

Figure 2 Effects of in vivo CMC2.24 treatment on MMPs and Resolvin $D_{1}$ in the elicited and non-elicited models of diabetic rat. (A) MMP-9 activities were analyzed by gelatin zymography and scanned by densitometer, and pro-MMP-9 levels were quantified by image J in the thioglycolate-elicited M $\varphi s$ conditioned media. (B) MMP-9 activities were analyzed by gelatin zymography, scanned by densitometer, and quantified by image $J$ in the elicited cell-free peritoneal fractions (CFPFs). (C) The pro-, active-, and total-MMP-9 activities were analyzed by gelatin zymography, scanned by densitometer, and quantified by image J in the non-elicited peritoneal CFPFs. (D) MMP-2 activities were analyzed by gelatin zymography and scanned by densitometer, and pro-MMP-2 levels were quantified by image J in the non-elicited peritoneal CFPFs. (E) RvD, levels ( $\mathrm{pg} / \mathrm{mL}$ ) in thioglycolate-elicited $M \varphi$ conditioned medium. (F) RvD, levels ( $\mathrm{pg} / \mathrm{mL})$ in non-elicited resident $\mathrm{M} \varphi$ conditioned medium. Each value represents Mean ( $n=6 /$ group) \pm Standard Error (S.E.M.). ${ }^{*} p<0.05$; ${ }^{*} p<0.005$, ${ }^{*}{ }^{*} p<0.00$, values were compared between groups at the same time period. Abbreviations: $\mathrm{N}$, normal group; $\mathrm{D}$, diabetic group; $\mathrm{D}+\mathrm{CMC2.24}$, diabetes+CMC2.24 treatment group. 


\section{Resolvins}

The thioglycolate-elicited peritoneal $\mathrm{M} \varphi$ s produced overall much higher levels of $\mathrm{RvD}_{1}$ (20-folds increase) than the non-elicited resident $\mathrm{M} \varphi \mathrm{s}$ (Figure $2 \mathrm{E}$ and $\mathrm{F}$ ). Diabetes significantly suppressed the $\mathrm{RvD}_{1}$ levels by $36 \%$ in elicited M $\varphi s$ (Figure 2E) and $86 \%$ in non-elicited $\mathrm{M} \varphi \mathrm{s}$ (Figure 2F) $(\mathrm{p}<0.005)$. CMC2.24 treatment of diabetics increased $\mathrm{RvD}_{1}$ levels by $29 \%$ in elicited $\mathrm{M} \varphi$ s (Figure 2E) and $84 \%$ in non-elicited $\mathrm{M} \varphi \mathrm{s}$ (Figure $2 \mathrm{~F})(\mathrm{p}<0.05$ ), restoring this resolvin in the diabetics essentially back to normal levels. The other resolvins, $\mathrm{RvE}_{1}$ and $\mathrm{LxA}_{4}$, were undetectable in both models of rat peritoneal M $\varphi s$.

\section{Diabetes-Induced Periodontitis and Osteoporosis \\ Alveolar Bone Loss}

In the elicited model, morphometric analyses showed a pattern consistent with diabetes-induced periodontitis. The severity of alveolar bone loss, as represented by an increase of the CEJ-ABC distance bucco-palatally (Figure $3 \mathrm{~A}$ ) on the distal side of the 1st molar were significantly greater $(\mathrm{p}<0.05)$ in $\mathrm{D}$ group than rats in $\mathrm{N}$ and $\mathrm{D}$ + CMC2.24 groups (Figure 3B). The lack of a significant difference in alveolar bone loss between the latter two groups $(\mathrm{p}>0.05)$, indicated that $\mathrm{CMC} 2.24$ treatment was able to
A

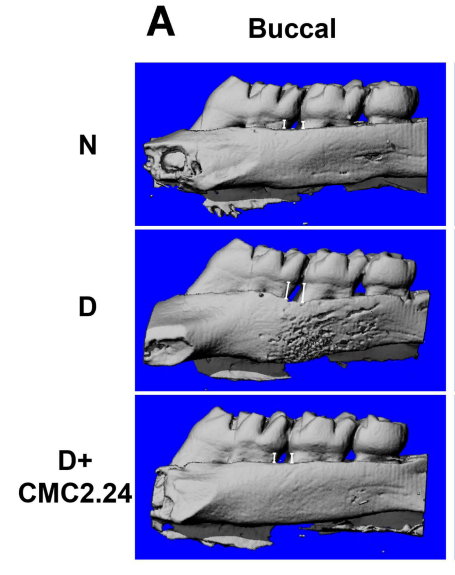

D

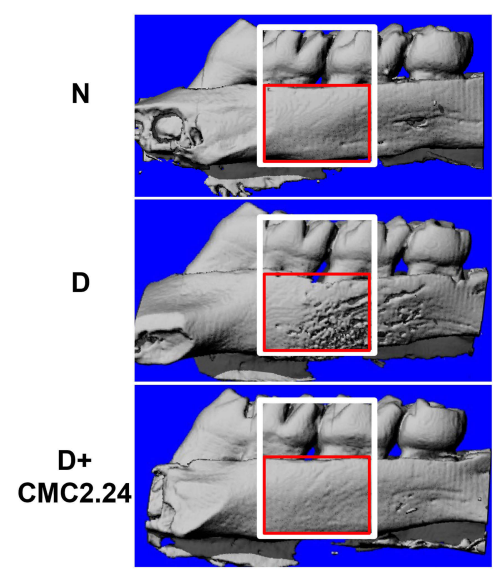

Palatal

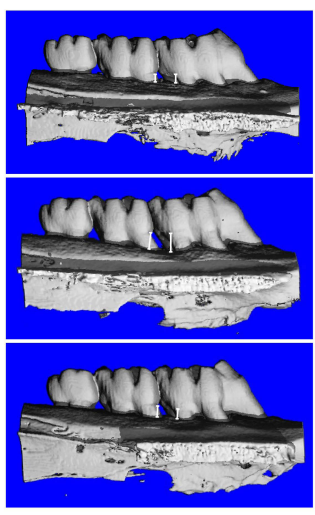

E

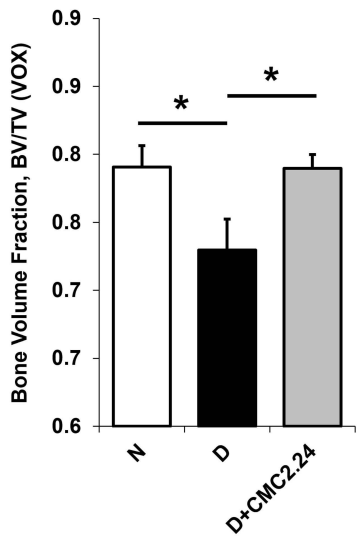

B

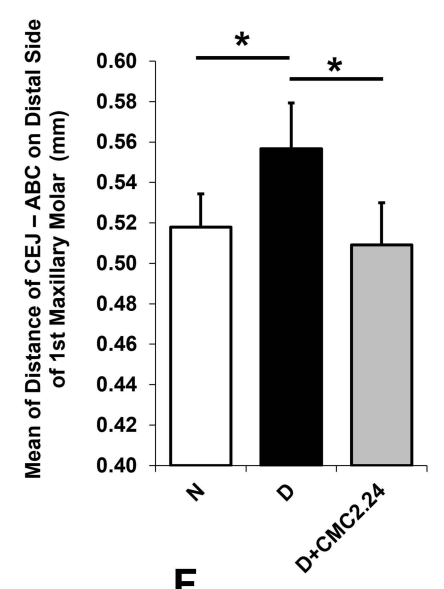

$\mathbf{F}$

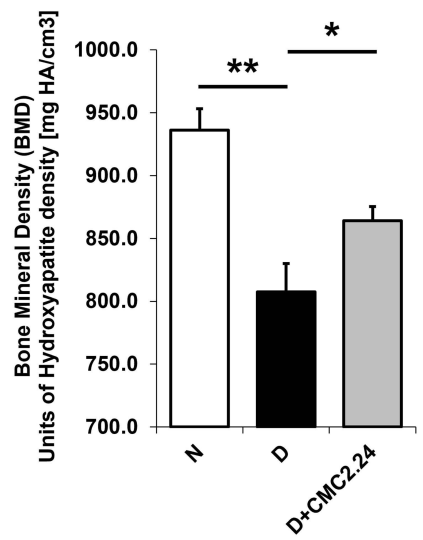

C
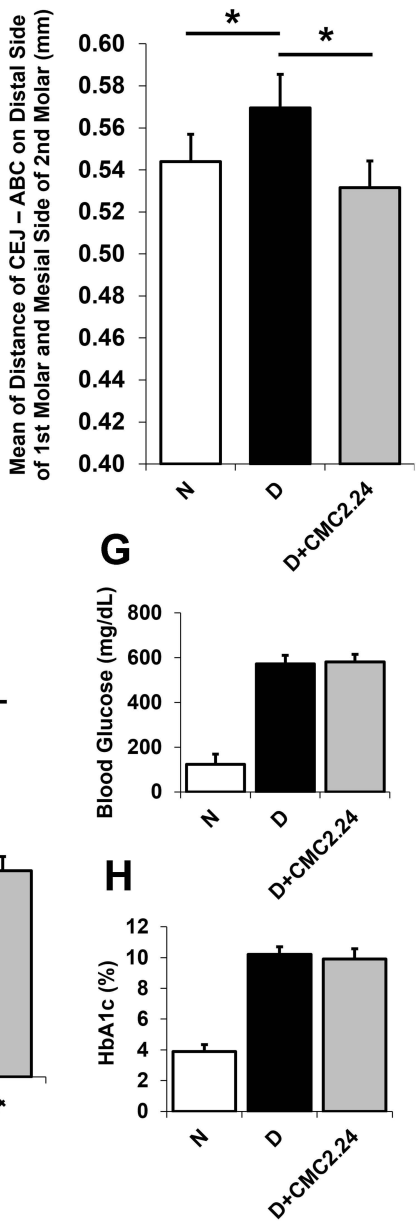

Figure 3 Morphometric analyses of diabetes-induced periodontal alveolar bone loss and osteoporosis measured by $\mu$ CT in rat with 4-day thioglycolation. (A) White "markers" (flat-end line, "I") indicates the distance from CEJ to ABC at the sites between the distal side of the Ist molar and the mesial side of the 2nd molar, bucco-palatally, representing alveolar bone loss. (B) Mean of distance $(\mathrm{mm})$ of CEJ-ABC at the distal side of the Ist maxillary molar on both buccal and palatal view. (C) Mean of distance (mm) of CEJ-ABC at both the distal side of the Ist molar and the mesial side of the 2 nd molar buccal-palatally. (D) Morphometric analyses of diabetes-induced osteoporosis measured by $\mu \mathrm{CT}$ in rat with 4-day thioglycolation. The total volume of interest (VOI) area indicates in the white box. And the bone volume of interest area indicates in the red box. (E) The analysis of bone volume fraction (BV/TV) by $\mu \mathrm{CT}$. (F) The analysis of bone mineral density (BMD) by $\mu \mathrm{CT}$ based on calculating the units of hydroxyapatite density $\left(\mathrm{mg} \mathrm{HA} / \mathrm{cm}^{3}\right)$. (G) The levels of blood glucose $(\mathrm{mg} / \mathrm{dL})$ in normal/control (non-diabetic), untreated diabetic and the $\mathrm{CMC} 2.24-$ treated diabetic rats, respectively. $(\mathbf{H})$ The levels of $\mathrm{HbAlc}(\%)$ in normal/control (non-diabetic), untreated diabetic and the $\mathrm{CMC2.24-treated} \mathrm{diabetic} \mathrm{rats,} \mathrm{respectively.} \mathrm{Each} \mathrm{value} \mathrm{represents} \mathrm{Mean} \mathrm{(} \mathrm{n}=6$ / group) \pm Standard Error (S.E.M.). ${ }^{*} \mathrm{p}<0.05$; ${ }^{* *} \mathrm{p}<0.005$, values were compared between groups at the same time period.

Abbreviations: VOX, based on counting voxels; TV, total volume $\left(\mathrm{mm}^{3}\right)$; BV, bone volume $\left(\mathrm{mm}^{3}\right)$; N, normal group; D, diabetic group; D+CMC2.24, diabetes+CMC2.24 treatment group. 
"normalize" excessive alveolar bone loss. Consistent with these results, the total levels of buccal-palatal alveolar bone loss on both the distal side of the 1 st molars and the mesial side of the 2nd molars were dramatically increased $(\mathrm{p}<0.05)$ in $\mathrm{D}$ group, compared to the values in $\mathrm{N}$ and $\mathrm{D}+\mathrm{CMC} 2.24$ groups (Figure 3C). Importantly, no significant difference of alveolar bone loss was detected between the latter two groups ( $p>0.05$ ), showing the efficacy of CMC2.24 treatment in preventing alveolar bone loss due to diabetes.

\section{Osteoporosis of Jawbone}

In the elicited model, diabetes-induced osteoporosis was observed in the jawbone. The 3D- $\mu \mathrm{CT}$ morphometric analyses of the maxilla showed the porosity in these bones was dramatically increased in untreated diabetics, compared to $\mathrm{N}$ and $\mathrm{D}+\mathrm{CMC} 2.24$ groups (Figure 3D). The latter two groups had no difference of porosity. This was confirmed by the analysis of bone volume fraction (BV/ TV): As shown in Figure 3D, BV and TV were indicated by the red and white boxes, respectively. $\mathrm{D}$ rats exhibited a significantly smaller percentage of BV/TV $(p<0.05)$, indicating the lower relative $\mathrm{BV}$ than $\mathrm{N}$ and $\mathrm{D}+\mathrm{CMC} 2.24$ rats (Figure 3E). CMC2.24 treatment increased $(\mathrm{p}<0.05)$ the relative $\mathrm{BV}$ back to essentially normal levels (Figure 3E). Moreover, analysis of BMD was consistent with the results of relative $\mathrm{BV}$, presenting a substantial reduction $(p<0.005)$ of $B M D$ in the untreated diabetics (Figure 3F), while the reduced BMD in the maxilla of diabetic rats was "rescued" by CMC2.24 treatment $(\mathrm{p}<$ $0.05)$, back to normal status ( $\mathrm{p}>0.05$ ) (Figure $3 \mathrm{~F})$.

\section{Blood Glucose and HbAlc}

The blood glucose and HbA1c levels were $600 \mathrm{mg} / \mathrm{dL}$ and $10 \%$ (Figure $3 \mathrm{G}$ and $\mathrm{H}$ ), respectively, in the untreated and CMC2.24-treated diabetic rats. Note that, for all of these beneficial changes produced by $\mathrm{CMC} 2.24$ treatment, no improvement was seen for blood glucose and HbA1c; $\mathrm{D}$ and $\mathrm{D}+\mathrm{CMC} 2.24$ rats were still severely hyperglycemic at the end of study, similar to our previous observation. ${ }^{27}$ However, in spite of the persistent hyperglycemia, there were no adverse events in $\mathrm{D}+\mathrm{CMC} 2.24$ group during the 3-week protocol, in contrast to the untreated diabetics which showed multiple complications such as tail necrosis and inflamed sclera of the eye.

\section{Discussion}

Research has shown that periodontitis is associated with other chronic systemic diseases, such as diabetes, ${ }^{32}$ osteoporosis, ${ }^{33}$ rheumatoid arthritis ${ }^{34}$ and coronary heart disease. $^{35}$ For a long time, it was thought that microbial biofilm was the factor that linked periodontitis to other diseases in the body; however, more recent research ${ }^{36}$ demonstrates that inflammation may be largely responsible for the association. Therefore, treating inflammation and modulating the host response may not only help manage periodontitis, but may also help with the management of other chronic inflammatory conditions. ${ }^{16,37-39}$

Recently, we identified the pleiotropic properties of a novel HMT compound, CMC2.24, in three rat models of experimental periodontitis. ${ }^{21,27,40}$ Its ability to "normalize" the inflammation/collagenolysis was also identified in oral and extraoral tissues. ${ }^{21,27,40}$ In the current report, we extend these studies of $\mathrm{CMC} 2.24$ to include a previously unrecognized ability of this phenylaminocarbonyl curcumin to upregulate resolvin activity and "normalize" immune cell functions, in addition to extending our earlier studies on normalization of pathologically-excessive cytokines and MMPs. Thus, we found that the function of macrophages which is suppressed by diabetes, was beneficially affected by CMC2.24's resolvin-like activity.

In brief, the impaired immune-cell function caused by hyperglycemia is associated with diabetes-induced complications, ${ }^{41}$ thus thioglycolate was used as a stimulus to induce an inflammatory response in the peritoneal cavity of diabetic rats. In the elicited model, diabetes led to an abnormally increased accumulation of macrophages in the peritoneal cavity. However, these cells exhibited impaired function characterized by: decreased chemotaxis, and increased production of both proinflammatory cytokines (IL-1 $\beta$, IL-6, and TNF- $\alpha$ ) and pathologically-elevated collagenolytic enzymes (MMP-2 and MMP-9). The therapeutic potential of CMC2.24's resolvin-like activity was observed in this study by "normalizing" cell function and restoring cell activity during inflammation. And its therapeutic potential is also supported by several recent publications which indicated: (a) a distinct and early increase in circulating MMP-9 in COVID-19 patients with respiratory failure, and demonstrated that only the plasma marker, MMP-9, correlated to neutrophil count and the severity of COVID-19 related pneumonia; ${ }^{6}$ and (b) the potential of using HMT (NONantibiotic doxycycline, developed and used initially for chronic inflammatory periodontitis) as a strategy for the prevention of ARDS, a deadly outcome of COVID-19. ${ }^{42}$

In addition, we also evaluated the resident macrophage cell function in the non-elicited model. Similar pathologic 
conditions in these cells were observed during diabetes, but at much lower levels compared to the levels under thioglycolate stimulation. Specifically, CMC2.24-treated diabetic rats showed a significant reduction of a key proinflammatory cytokine, IL- $1 \beta$, in the non-elicited resident macrophage. Moreover, data indicated that in the rPW, pathologically-elevated activities of two gelatinases (MMP9 and MMP-2) were also "normalized" by CMC2.24 treatment in the diabetic rats.

Of importance, this study describes a significant increase in resolvin $\left(\mathrm{RvD}_{1}\right)$ production in both non-elicited and elicited macrophages from the diabetic rats treated with $\mathrm{CMC} 2.24$ in vivo, and is consistent with our previous observations in a macrophage cell culture study. ${ }^{43}$ This important lipid mediator is derived from DHA, and exhibits a potent ability to resolve acute inflammation, thus preventing its prolongation. ${ }^{44}$ Studies have shown that $\mathrm{RvD}_{1}$ involves the inactivation of glycogen synthase kinase 3 beta (GSK3 $\beta$ ), a serine/threonine protein kinase involved in inflammation, simultaneously augmentation of GSK3 $\beta$ anti-inflammatory axis, and thus enhances resolution in LPS-stimulated/TLR4-engaged primary human monocytes. ${ }^{45}$ Although $\mathrm{RvD}_{1}$ has usually been associated with resolution of polymorphonuclear leukocyte infiltration/migration during acute inflammation, ${ }^{46,47}$ our studies indicate that $\mathrm{RvD}_{1}$ can also be produced by macrophages both in cell culture, and in animals, to resolve chronic inflammation, and was significantly increased by CMC2.24 treatment. Moreover, the enhanced activity of $\mathrm{RvD}_{1}$ seems to be the "targeted resolvin" of CMC2.24 treatment. The other major resolvins such as $\mathrm{RvE}_{1}$ and $\mathrm{LxA}_{4}$ have less sensitivity to $\mathrm{CMC} 2.24$ treatment, compared to $\mathrm{RvD}_{1}$. More mechanisms can be further explored in future studies.

Regarding broad therapeutic potential, we also observed a potent ability of CMC2.24 to reduce diabetesinduced bone height loss in alveolar bone, and diabetesinduced osteoporosis in rat jaws. This complication in the rats with severe hyperglycemia was characterized by increased roughness and porosity of the maxilla based on 3D-reconstructed images, and further confirmed by $\mu \mathrm{CT}$ morphometric analysis of BV and BMD analysis. These data demonstrated no significant difference between the CMC2.24-treated diabetic rats and non-diabetics, indicating that $\mathrm{CMC} 2.24$ treatment prevents local and systemic bone loss (diabetes-induced periodontitis and osteoporosis). However, whether treatment with $\mathrm{CMC} 2.24$ can reduce the risk for pathologic fracture of skeletal bones in poorly controlled diabetics also remains to be demonstrated in future studies.
Notably, all these beneficial responses of diabetic rats to $\mathrm{CMC} 2.24$ occurred without any reduction in the severity of hyperglycemia. This observation suggests the potential of $\mathrm{CMC} 2.24$ treatment for reducing serious diabetic complications, even during uncontrolled hyperglycemia, which is supported by the lack of adverse events in the CMC2.24-treated diabetics at least during the 3-weeks protocol.

\section{Conclusion}

In conclusion, the current study indicates that in vivo administration of CMC2.24 to diabetic rats "normalizes" inflammatory (and other) cell functions as shown by: (a) decreasing the abnormal accumulation of macrophages in the peritoneal cavity; (b) improving inflammatory cell chemotactic activity; (c) significantly suppressing both pathologically-elevated levels of MMPs and their activation; (d) attenuating the severity of systemic inflammation as indicated by reduced pro-inflammatory cytokines in peritoneal macrophages (exvivo); and (e) significantly enhancing resolvin $\left(\mathrm{RvD}_{1}\right)$ activity. These beneficial effects ultimately contributed, at least in part, to the reduction of local alveolar bone loss (diabetesinduced periodontitis), and the severity of systemic bone loss (diabetes-induced osteoporosis). These anti-collagenolytic (MMP-inhibition), anti-inflammatory, and pro-resolvin benefits occurred without any effect on severe hyperglycemia, and no adverse events were observed in the CMC2.24-treated diabetic rats, strongly indicating the therapeutic potential of this novel compound even in uncontrolled diabetics. Lastly, pharmacokinetic and safety studies ${ }^{48}$ are being carried out in different animal models, which is necessary before this compound can be considered for testing in human clinical trials.

\section{Data Sharing Statement}

All other supporting information is available upon request from the corresponding author.

\section{Acknowledgments}

This study was funded by NIDCR grant [R41 DE024946], NYSTAR [A43273] and Chem-Master Intl., Inc. The authors of this study thank the veterinary and technical personnel of the Division of Laboratory Animal Resources (DLAR) for assistance during animal handling and experimental procedures. The authors also thank Peggy Yang (Oral Biology and Pathology, Stony Brook University, NY, USA) for assistances with the rat study; Dindo Q. Mijares (Biomaterials, New York University College of Dentistry, NY, USA) for the $\mu \mathrm{CT}$ scanning and data 
acquisition; Xiaofei Li, and Yi-Xian Qin (Department of Biomedical Engineering, Stony Brook University, NY, USA) for the $\mu \mathrm{CT}$ data acquisition.

\section{Disclosure}

LM Golub is listed as an inventor on several related patents, including CMC2.24, and these have been fully assigned to his institution, Stony Brook University, The State University of New York (SUNY). F Johnson is also listed as an inventor on several related patents, including CMC2.24, which have been fully assigned to Stony Brook University and to ChemMaster Int. Inc., on a shared basis. In addition, Traverse Biosciences Inc. has exclusively licensed patents from the Research Foundation for the State University of New York (RF/SUNY) covering the structure and use of the chemically modified curcumins for the purpose of commercialization in human and animal health. The remaining authors declare that the research was conducted in the absence of any commercial or financial relationships that could be construed as a potential conflict of interest.

\section{References}

1. Isola G, Polizzi A, Santonocito S, Alibrandi A, Williams RC. Periodontitis activates the NLRP3 inflammasome in serum and saliva. J Periodontol. 2021. doi:10.1002/JPER.21-0049

2. Isola G, Lo Giudice A, Polizzi A, Alibrandi A, Murabito P, Indelicato F. Identification of the different salivary Interleukin-6 profiles in patients with periodontitis: a cross-sectional study. Arch Oral Biol. 2021;122:104997. doi:10.1016/j.archoralbio.2020.104997

3. Iacopino AM. Periodontitis and diabetes interrelationships: role of inflammation. Ann Periodontol. 2001;6(1):125-137. doi:10.1902/ annals.2001.6.1.125

4. Kinane DF, Stathopoulou PG, Papapanou PN. Periodontal diseases. Nat Rev Dis Primers. 2017;3:17038. doi:10.1038/nrdp.2017.38

5. Golub LM, Payne JB, Reinhardt RA, Nieman G. Can systemic diseases co-induce (not just exacerbate) periodontitis? A hypothetical "two-hit" model. J Dent Res. 2006;85(2):102-105. doi:10.1177/154405910608500201

6. Ueland T, Holter JC, Holten AR, et al. Distinct and early increase in circulating MMP-9 in COVID-19 patients with respiratory failure. $J$ Infect. 2020;81(3):e41-e43. doi:10.1016/j.jinf.2020.06.061

7. Abers MS, Delmonte OM, Ricotta EE, et al. An immune-based biomarker signature is associated with mortality in COVID-19 patients. JCI Insight. 2021;6:1. doi:10.1172/jci.insight.144455

8. Frankwich K, Tibble C, Torres-Gonzalez M, et al. Proof of Concept: matrix metalloproteinase inhibitor decreases inflammation and improves muscle insulin sensitivity in people with type 2 diabetes. J Inflamm. 2012;9(1):35. doi:10.1186/1476-9255-9-35

9. Derosa G, Ferrari I, D'Angelo A, et al. Matrix metalloproteinase-2 and -9 levels in obese patients. Endothelium. 2008;15(4):219-224. doi:10.1080/10623320802228815

10. Saini R, Saini S, Sugandha R. Periodontal disease: the sixth complication of diabetes. J Family Community Med. 2011;18(1):31. doi:10.4103/1319-1683.78636

11. Cairo F, Rotundo R, Frazzingaro G, Muzzi L, Pini Prato GP. Diabetes mellitus as a risk factor for periodontitis. Minerva Stomatol. 2001;50 (9-10):321-330.
12. Golub LM, Lee HM, Lehrer G, et al. Minocycline reduces gingival collagenolytic activity during diabetes. Preliminary observations and a proposed new mechanism of action. J Periodontal Res. 1983;18 (5):516-526. doi:10.1111/j.1600-0765.1983.tb00388.x

13. Golub LM, Elburki MS, Walker C, et al. Non-antibacterial tetracycline formulations: host-modulators in the treatment of periodontitis and relevant systemic diseases. Int Dent J. 2016;66(3):127-135. doi:10.1111/ idj.12221

14. Payne JB, Golub LM, Stoner JA, et al. The effect of subantimicrobial-dose-doxycycline periodontal therapy on serum biomarkers of systemic inflammation: a randomized, double-masked, placebo-controlled clinical trial. J Am Dent Assoc. 2011;142 (3):262-273. doi:10.14219/jada.archive.2011.0165

15. Gu Y, Walker C, Ryan ME, Payne JB, Golub LM. Non-antibacterial tetracycline formulations: clinical applications in dentistry and medicine. J Oral Microbiol. 2012;1:4.

16. Golub LM, Lee HM. Periodontal therapeutics: current host-modulation agents and future directions. Periodontol 2000. 2020;82(1):186-204. doi:10.1111/prd.12315

17. Serhan CN. Systems approach with inflammatory exudates uncovers novel anti-inflammatory and pro-resolving mediators. Prostaglandins Leukot Essent Fatty Acids. 2008;79(3-5):157-163. doi:10.1016/j. plefa.2008.09.012

18. Van Dyke TE, Hasturk H, Kantarci A, et al. Proresolving nanomedicines activate bone regeneration in periodontitis. J Dent Res. 2015;94 (1):148-156. doi:10.1177/0022034514557331

19. Zhang Y, Gu Y, Lee HM, et al. Design, synthesis and biological activity of new polyenolic inhibitors of matrix metalloproteinases: a focus on chemically-modified curcumins. Curr Med Chem. 2012;19 (25):4348-4358. doi:10.2174/092986712802884295

20. Zhang Y, Golub LM, Johnson F, Wishnia A. pKa, zinc- and serum albumin-binding of curcumin and two novel biologically-active chemically-modified curcumins. Curr Med Chem. 2012;19 (25):4367-4375. doi:10.2174/092986712802884240

21. Elburki MS, Rossa C, Guimaraes MR, et al. A novel chemically modified curcumin reduces severity of experimental periodontal disease in rats: initial observations. Mediators Inflamm. 2014;2014:959471. doi:10.1155/2014/959471

22. Deng J, Golub LM, Lee HM, et al. Chemically-Modified Curcumin 2.24: a Novel Systemic Therapy for Natural Periodontitis in Dogs. $J$ Exp Pharmacol. 2020;12:47-60. doi:10.2147/JEP.S236792

23. Zhang Y, McClain SA, Lee HM, et al. A Novel Chemically Modified Curcumin "Normalizes" Wound-Healing in Rats with Experimentally Induced Type I Diabetes: initial Studies. $J$ Diabetes Res. 2016;2016:5782904. doi:10.1155/2016/5782904

24. Wang HH, Lee HM, Raja V, et al. Enhanced efficacy of chemically modified curcumin in experimental periodontitis: systemic implications. J Exp Pharmacol. 2019;11:1-14. doi:10.2147/JEP.S171119

25. Katzap E, Goldstein MJ, Shah NV, et al. The chondroprotective capability of curcumin (curcuma longa) and its derivatives against IL-1 $\beta$ and OsM-mediated chrondrolysis. Trans Orthoped Res Soc. 2011;36(Speciallssue 1707):59.

26. Mallangada NA, Vargas JM, Thomas S, et al. A novel tricarbonylmethane agent (CMC2.24) reduces human pancreatic tumor growth in mice by targeting Ras. Mol Carcinog. 2018;57(9):1130-1143. doi:10.1002/mc.22830

27. Elburki MS, Moore DD, Terezakis NG, et al. A novel chemically modified curcumin reduces inflammation-mediated connective tissue breakdown in a rat model of diabetes: periodontal and systemic effects. J Periodontal Res. 2017;52(2):186-200. doi:10.1111/ jre. 12381

28. Misharin AV, Saber R, Perlman H. Eosinophil contamination of thioglycollate-elicited peritoneal macrophage cultures skews the functional readouts of in vitro assays. J Leukoc Biol. 2012;92 (2):325-331. doi:10.1189/jlb.1111560 
29. Park CH, Abramson ZR, Taba M Jr, et al. Three-dimensional micro-computed tomographic imaging of alveolar bone in experimental bone loss or repair. J Periodontol. 2007;78(2):273-281. doi:10.1902/jop.2007.060252

30. Cengiz IF, Oliveira JM, Reis RL. Micro-CT - a digital 3D microstructural voyage into scaffolds: a systematic review of the reported methods and results. Biomater Res. 2018;22:26. doi:10.1186/s40824018-0136-8

31. Liao G, Simone J, Simon SR. Paracrine downregulation of Fc gamma RIII in human monocyte-derived macrophages induced by phagocytosis of nonopsonized particles. Blood. 1994;83(8):2294-2304. doi:10.1182/blood.V83.8.2294.2294

32. Nishimura F, Kono T, Fujimoto C, Iwamoto Y, Murayama Y. Negative effects of chronic inflammatory periodontal disease on diabetes mellitus. J Int Acad Periodontol. 2000;2(2):49-55.

33. Golub LM, Ramamurthy NS, Llavaneras A, et al. A chemically modified nonantimicrobial tetracycline (CMT-8) inhibits gingival matrix metalloproteinases, periodontal breakdown, and extra-oral bone loss in ovariectomized rats. Ann $N$ Y Acad Sci. 1999;878:290-310. doi:10.1111/j.1749-6632.1999.tb07691.x

34. Bartold PM, Lopez-Oliva I. Periodontitis and rheumatoid arthritis: an update 2012-2017. Periodontol. 2020;83(1):189-212. doi:10.1111/ prd. 12300

35. Isola G, Polizzi A, Alibrandi A, Williams RC, Lo Giudice A. Analysis of galectin-3 levels as a source of coronary heart disease risk during periodontitis. J Periodontal Res. 2021;56(3):597-605. doi:10.1111/jre. 12860

36. Williams RC. A century of progress in understanding periodontal disease. Compend Contin Educ Dent. 2002;23(5 Suppl):3-10.

37. Preshaw PM. Host response modulation in periodontics. Periodontol 2000. 2008;48:92-110. doi:10.1111/j.1600-0757.2008.00252.x

38. Preshaw PM. Host modulation therapy with anti-inflammatory agents. Periodontol 2000. 2018;76(1):131-149. doi:10.1111/ prd. 12148

39. Golub LM, Ryan ME, Williams RC. Modulation of the host response in the treatment of periodontitis. Dent Today. 1998;17(10):102-106, 108-109.
40. Elburki MS, Rossa C Jr, Guimaraes-Stabili MR, et al. A Chemically Modified Curcumin (CMC 2.24) Inhibits Nuclear Factor kappaB Activation and Inflammatory Bone Loss in Murine Models of LPS-Induced Experimental Periodontitis and Diabetes-Associated Natural Periodontitis. Inflammation. 2017;40(4):1436-1449. doi:10.1007/s10753-017-0587-4

41. Ratter JM, Tack CJ, Netea MG, Stienstra R. Environmental Signals Influencing Myeloid Cell Metabolism and Function in Diabetes. Trends Endocrinol Metab. 2018;29(7):468-480. doi:10.1016/j. tem.2018.04.008

42. Cardoso EOC, Fine N, Glogauer M, et al. The Advent of COVID-19; Periodontal Research Has Identified Therapeutic Targets for Severe Respiratory Disease; an Example of Parallel Biomedical Research Agendas. Front Dent Med. 2021;2.

43. Deng J, Gu Y, Lee HM, Raja V, Johnson F, Golub LM. Novel Modified-Curcumin: resolution of Cytokines and MMPs In Cell Culture. J Dent Res. 2018;97(SpecialIssue A):0129.

44. Yin Y, Chen F, Wang W, Wang H, Zhang X. Resolvin D1 inhibits inflammatory response in STZ-induced diabetic retinopathy rats: possible involvement of NLRP3 inflammasome and NF-kappaB signaling pathway. Mol Vis. 2017;23:242-250.

45. Gu Z, Lamont GJ, Lamont RJ, Uriarte SM, Wang H, Scott DA. Resolvin D1, resolvin D2 and maresin 1 activate the GSK3beta anti-inflammatory axis in TLR4-engaged human monocytes. Innate Immun. 2016;22(3):186-195. doi:10.1177/1753425916628618

46. Serhan CN, Hong S, Gronert K, et al. Resolvins: a family of bioactive products of omega-3 fatty acid transformation circuits initiated by aspirin treatment that counter proinflammation signals. J Exp Med. 2002;196(8):1025-1037. doi:10.1084/jem.20020760

47. Serhan CN, Chiang N. Resolution phase lipid mediators of inflammation: agonists of resolution. Curr Opin Pharmacol. 2013;13 (4):632-640. doi:10.1016/j.coph.2013.05.012

48. Bhatt HD, McClain S, Lee HM, et al. The Maximum-Tolerated-Dose And Pharmacokinetics Of A Novel Chemically-Modified-Curcumin In Rats. J Dent Res. 2020;99(Speciallssue A):3665.
Journal of Inflammation Research

\section{Publish your work in this journal}

The Journal of Inflammation Research is an international, peerreviewed open-access journal that welcomes laboratory and clinica findings on the molecular basis, cell biology and pharmacology of inflammation including original research, reviews, symposium reports, hypothesis formation and commentaries on: acute/chronic inflammation; mediators of inflammation; cellular processes; molecular mechanisms; pharmacology and novel anti-inflammatory drugs; clinical conditions involving inflammation. The manuscript management system is completely online and includes a very quick and fair peerreview system. Visit http://www.dovepress.com/testimonials.php to read real quotes from published authors. 